\title{
Investigation of water to ice phase change in porous media by ultrasonic and dielectric measurements
}

\author{
Antonin Fabbri ${ }^{1,2}$, Teddy Fen-Chong ${ }^{3}$, Aza Azouni ${ }^{4}$, Jean-François Thimus ${ }^{5}$
}

\begin{abstract}
The main objective of this paper is to study the evolution of the ice content of porous media submitted to sub-zero temperatures by dielectric and ultrasonic measurements. Dielectric measurements are made by a capacitive sensor-based apparatus. The amount of ice formed within the tested sample is estimated from the global dielectric constants of the sample and of all the phases that form the tested composite material. On the other hand, ultrasonic measurements are based on the evolution of the ultrasonic wave velocity through the tested sample during a freezing-thawing cycle. These two methods lead to very close results and appear to be cheaper alternatives to low temperature calorimetry. The ice content curves are analyzed with the help of thermoporometry concepts in order to characterize the pore size distribution. Results appear to be complementary to mercury intrusion porosimetry ones. Moreover the commonly observed hysteresis of the ice content during a freezing-thawing cycle is investigated with respect to material microstructure.
\end{abstract}

Keywords: Freeze-thaw; Pore size distribution; Hysteresis; Cement; Clay; Thermodynamics

\section{INTRODUCTION}

Damage induced by frost action upon concrete structures is a source of main concern in cold climates (Pigeon 1984; Dash et al. 1995). The mechanical response of a porous mate-

\footnotetext{
${ }^{1} \mathrm{PhD}$, Université Paris-Est, Institut Navier, LMSGC 2 allée Kepler - 77420 Champs-sur-Marne, France.

${ }^{2}$ New Address: BRGM-GEO, 3 avenue Claude Guillemin, BP 6009 - 45060 Orléans cedex 2, France.

${ }^{3}$ Research Fellow, Université Paris-Est, Institut Navier, LMSGC 2 allée Kepler - 77420 Champs-surMarne, France.

${ }^{4}$ CNRS Research Director, Université Paris-Est, Institut Navier, LMSGC 2 allée Kepler - 77420 Champssur-Marne, France.

${ }^{5}$ Unité de Génie Civil et Environnemental, Université Catholique de Louvain, B-1348 Louvain-la-Neuve, Belgique.
} 
rial is credited to result from the combination of the liquid-solid expansion, the transport of unfrozen liquid water through the porous network, and the presence of air voids (Wang et al. 1996). Theoretical approaches have been recently developed to understand and quantify the phenomena at the pore scale (Coussy and Fen-Chong 2005), at the material scale (Coussy 2005) and at the structure scale (Fabbri et al. 2008). Whatever the approach carried out, the volumetric ratio of frozen water (commonly called the ice saturation ratio, $S_{c}$ ) versus temperature turns out to be the key curve governing the mechanical behavior of porous materials upon frost action. Freezing of cement based materials is usually studied with low temperature calorimetry (Bager and Sellevold 1986). However this device only allows to test very small samples (characteristic length round the millimeter) which are often crushed. Hence tests on non-crushed heterogeneous materials like mortar and concrete (aggregate characteristic length around the centimeter) should be difficult to set up. For such materials requiring larger samples, dielectric (Fabbri et al. 2006) and ultrasonic (Thimus et al. 1993) methods are more appropriate while being less expensive. This paper investigates these two methods and compares the results they provide on the ice / water transition in cohesive porous materials such as cement pastes. Then the hysteresis on ice content commonly observed during a freezing-thawing cycle is investigated in relation with the material microstructure.

\section{BASIC MEASUREMENT PRINCIPLE}

\section{Ultrasonic method}

Ultrasonic methods rely on the difference between the longitudinal compression wave velocities in liquid water $\left(V_{l}=1428 \mathrm{~m} / \mathrm{s}\right.$ at $\left.0^{\circ} \mathrm{C}\right)$ and ice crystal $\left(V_{c}=3844 \mathrm{~m} / \mathrm{s}\right.$ at $\left.0^{\circ} \mathrm{C}\right)$ to evaluate the amount of unfrozen water in a saturated partially frozen porous medium. After a review of some classical schemes used in ultrasonic experiments (Thimus et al. 1991; Thimus et al. 1993), the link between the wave velocity through a partially frozen porous sample $(V)$ and the volumetric ratio of ice $\left(S_{c}\right)$, is established by the Timur model (Timur 
1968):

$$
\frac{1}{V}=\frac{\phi_{0}\left(1-S_{c}\right)}{V_{l}}+\frac{\phi_{0} S_{c}}{V_{c}}+\frac{1-\phi_{0}}{V_{s}}
$$

where $V_{s}$ is the longitudinal compression wave velocity in the solid matrix and $\phi_{0}$ the porosity of the porous medium.

The relation (1) leads to the following expression in which $S_{c}$ is directly linked to $V$ :

$$
S_{c}=\frac{a}{V}+b
$$

where $a=V_{c} V_{l} /\left(\phi_{0}\left(V_{l}-V_{c}\right)\right)$ and $b=-V_{c} V_{l} /\left(\phi_{0}\left(V_{l}-V_{c}\right)\right)\left(\left(1-\phi_{0}\right) / V_{s}+\phi_{0} / V_{l}\right)$.

However a great dispersion on $V_{l}$ and $V_{c}$ measurements on the same kind of sample has been observed. In fact these two parameters seem to strongly depend on material cure, age and sampling. In consequence, in this study, the coefficients $a$ and $b$ are fitted from the totally frozen $\left(S_{l}=0\right)$ and unfrozen $\left(S_{l}=1\right)$ states so that:

$$
\begin{aligned}
& a=\frac{V\left(S_{c}=0\right) V\left(S_{c}=1\right)}{V\left(S_{c}=0\right)-V\left(S_{c}=1\right)} \\
& b=\frac{V\left(S_{c}=1\right)}{V\left(S_{c}=1\right)-V\left(S_{c}=0\right)}=-\frac{a}{V\left(S_{c}=0\right)}
\end{aligned}
$$

\section{Capacitive method}

The capacitive method is based on the contrast between water $\left(\varepsilon_{l}\right.$ about 80 at $\left.20^{\circ} \mathrm{C}\right)$ and ice $\left(\varepsilon_{c}\right.$ about 3 between $-40^{\circ} \mathrm{C}$ and $\left.0^{\circ} \mathrm{C}\right)$ dielectric constants in the radio-frequency range (Fen-Chong et al. 2004; Fen-Chong and Fabbri 2005; Fabbri et al. 2006). In consequence, any solidification of the porous network liquid will induce a notable decrease of the global dielectric constant of the sample $(\varepsilon)$. To get the freezing curve, a relation between $\varepsilon$ and the ice saturation ratio $S_{c}$ must be established. To do so, after a review of some classical mixture schemes (Zakri et al. 1998; Fen-Chong et al. 2004), the Lichtenecker model was

chosen. This model comes from the Effective Medium Theory in which the self-consistent 
scheme is adopted. This finally gives (Fabbri et al. 2006)):

$$
\varepsilon=\varepsilon_{m}^{1-\phi_{0}}\left(\left(1-S_{c}\right) \varepsilon_{l}^{\phi_{0}}+S_{c} \varepsilon_{c}^{\phi_{0}}\right)
$$

where $\varepsilon_{m}$ is the dielectric constant of the solid matrix. Whereas $\varepsilon_{c}$ is well known and remains constant during a freezing-thawing cycle, $\varepsilon_{l}$ varies with the temperature. In addition, the dielectric constant of the hydration water (Mercury et al. 2001) that is part of the mineral matrix, changes with temperature, which significantly affects $\varepsilon_{m}$.

Therefore, a semi-empirical method has been developped (Fabbri et al. 2006). It is based on the experimental determination of the dielectric constant of the solid matrix $\varepsilon_{m}$ in a dried sample (no capillary water) and that of the liquid water $\varepsilon_{l, s}$ for the same sample but in fully saturated conditions. The dielectric constant of the unfrozen water has been experimentally found to be obtained by extrapolating the affine $\varepsilon_{l, s}(T)$ relation. By doing so, one gets:

$$
\varepsilon_{m}(T)=\sqrt[1-\phi_{0}]{\frac{\varepsilon_{d r i e d}(T)}{\varepsilon_{a}^{\phi_{0}}}}
$$

where $\varepsilon_{a}=1$ is the dielectric constant of air, and

$$
\varepsilon_{l}(T)=\sqrt[\phi_{0}]{\frac{\varepsilon\left(S_{c}=0, T\right)}{\varepsilon_{m}^{1-\phi_{0}}(T)}}
$$

Using (5) and (6), (4) can be eventually inverted in the form:

$$
S_{c}=A(T) \varepsilon+B(T)
$$

where $1 / A=\varepsilon_{m}^{1-\phi_{0}}\left(\varepsilon_{c}^{\phi_{0}}-\varepsilon_{l}^{\phi_{0}}\right)$ and $B=-\varepsilon_{l}^{\phi_{0}} /\left(\varepsilon_{c}^{\phi_{0}}-\varepsilon_{l}^{\phi_{0}}\right)$.

\section{MATERIALS AND RELATED PROPERTIES}

In this study we used the following materials.

Sintered glass beads (SBG): Fused glass beads are made from commercial CVp silica 
glass powders which are between 62 to $87 \mu \mathrm{m}$ in diameter. The as-received materials are poured into a female mould which is then heated in an oven at $630^{\circ} \mathrm{C}$. In this way the beads collapse and fuse together to yield a cohesive porous medium. Mercury injection measurements show monodisperse porous distribution centred around $30 \mu \mathrm{m}$. Cylindrical samples of 50-mm mean diameter and 20-mm mean thickness were used.

Boom Clay (BC): The Boom Clay used in this study is sampled from the "Centre d'Etude Nucleaire" excavation site in Belgium at 200-m deep. Its general structure is a thin sheet stacking and its mineralogy is alike that given in (Romero et al. 1999). From the analysis of its microstructure by SEM (Dehandschutter et al. 2004) and Mercury Intrusion experiment (figure 1), Boom Clay appears to be a lightly consolidated medium with an important open porosity and composed of particles lined up with the stratigraphy. Three porosity peaks are identified on the pore diameter distribution : $150 \mathrm{~nm}, 2 \mu \mathrm{m}$ and $100 \mu \mathrm{m}$. The $300 \mu \mathrm{m}$ peak is attributed to the presence of cracks on the sample surface while the $2 \mu \mathrm{m}$ and $150 \mathrm{~nm}$-peaks are attributed to the inter-aggregate pore modes (Romero et al. 1999). Cylindrical samples of 60-mm mean diameter and 25-mm mean thickness were used.

Hardened cement paste (HCP): Cement paste with water-cement ratio of 0.5 are prepared with a 5-liter mortar mixer and cast in cylinder moulds with a 40-mm in diameter and 100-mm high. Ordinary Portland Cement CEM I CPA 52.5 N CP2 and distilled water are used. The porous distribution got from Mercury Intrusion Experiments is reported on figure 1 where the two observed peaks correspond to capillary porosity (100-nm in diameter) and inter-hydrates porosity ( 4-nm in diameter) (Baroghel-Bouny and Chaussadent 1993). One day after casting, samples are removed from their mould and stored in moist condition (relative humidity $r h=95 \% \pm 5 \%$ ) during 6 months. Then they are cut in about $25-\mathrm{mm}$ slices before being put in water until testing. Samples were saturated with degassed distilled water at $3 \mathrm{kPa}$ air pressure and tested.

Table 1 gives the tested materials main characteristics which are their global water porosity $\left(\phi_{0}\right)$, initial water content $(\omega)$, mean pore diameters $\left(D_{p}\right)$, density of the dried material 
$\left(\gamma_{d}\right)$, ultrasonic coefficients $a$ and $b$ (see (2)) and dielectric constants defined in (5) and (6). The fitted values from ultrasonic and capacitive experiments of $a, b, \varepsilon_{m}$, and $\varepsilon_{l}$ empirically take account of the mineralogy, microstructure of the porous media, as well as the interfacial polarization effects at stake in clays and cement materials. Indeed one can see on figure 2 that the dielectric constant of the in-pore water in SGB is alike that of bulk water (Ellison et al. 1996; Kaatze 1997; Fabbri 2006) whereas this is not the case for HCP and more importantly for BC. At last figure 3 indicates that the dielectric constant of the mineral solid in HCP is much higher than those in SGB and BC, which results from the presence of water molecules inside the C-S-H gel phase of cementitious materials.

\section{EXPERIMENTS AND RESULTS}

For each type of material the ice saturation ratio was evaluated at least four times: twice with the ultrasonic apparatus and twice with the capacitive method. The measurement sensitivity on $S_{c}$ is estimated to be around 0.01 for the two apparatuses (Thimus et al. 1993; Fabbri et al. 2006).

For ultrasonic experiments two samples are used. The first one is inserted into two transducers of 50-mm in diameter (an emitter and a receiver). Electric impulses (100 Volts pulse amplitude and 3 ps pulse width at a rate of 40 pulses per second) were generated by a PUNDIT unit (portable ultrasonic non-destructive digital indicating tester) and transformed into ultrasonic longitudinal compression waves in the emitter. After propagation through the sample, the signals were recorded by the receiver and treated by a numerical oscilloscope and a $\mathrm{PC}$ at a sampling rate of $2 \mathrm{MHz}$. The wave is supposed to be received by the receptor when the recorded amplitude reached $10 \%$ of the first peak value. Then its velocity is calculated by the ratio between the sample thickness and the delay of the wave through the sample. At the interface between the transducers and the sample, grease was used as a coupling agent to improve wave transmission (Couvreur and Thimus 1996). The second sample is drilled to insert a T-type thermocouple and to get the sample temperature history for a given imposed temperature time-evolution. The two samples are put in a $50 \mathrm{~cm} \times 50 \mathrm{~cm} \times 40 \mathrm{~cm}$ thermally 
insulated box connected to a liquid nitrogen reservoir. The temperature rate is $20^{\circ} \mathrm{C} /$ hour. Freezing is stopped at $-80^{\circ} \mathrm{C}$ and thawing at $20^{\circ} \mathrm{C}$. A one hour temperature step is made at the end of freezing (figure 5). Such an important freezing rate is chosen in order to reach quite rapidly the totally frozen state of the tested sample. A schematic representation of the ultrasonic device is shown in figure 4a.

For the capacitive experiments, the sample is inserted between two $60 \mathrm{~mm}$ diameter stainless steel electrodes connected to a $30 \mathrm{MHz}-50 \mathrm{MHz}$ oscillator electronic device. All of them form an oscillating electric circuit. Then, the dielectric constant of the sample $(\varepsilon)$ which is linked to the resonant frequency of the oscillating circuit, is measured. The temperature is imposed by a Galden PFPE HT200 cryogenic fluid (from Solvay Solexis) which circulates from a computer-controlled Hueber cryostat and measured by a T-type thermocouple inserted into the sample core. Likewise, in the Ultrasonic experiments the temperature rate is $20^{\circ} \mathrm{C}$ /hour. Freezing is stopped at $-40^{\circ} \mathrm{C}$ for the SGB and $\mathrm{BC}$ experiments and et $-30^{\circ} \mathrm{C}$ for the HCP experiments. Thawing is stopped at $0.1^{\circ} \mathrm{C}$. A two hours temperature step is made at the end of freezing (figure 5). The thermal insulation of the lateral surface is achieved by an expanded polystyrene annulus. In order to avoid surface desaturation during freezingthawing cycles, each sample is wrapped by a moisture resistant Parafilm sheet. A schematic representation of the capacitive device is shown in figure $4 \mathrm{~b}$.

A comparison of the temperature developed in the core of the sample in the two methods is shown in figure 5. During the freezing stage, the temperature of the sample follows the same pattern in both experimental devices used. It is then possible to compare the formation of ice in a cooling sample by the two devices without any effects from cooling rate differences. But, this is not the case of thawing where the heating rate is significantly higher during ultrasonic measurements. Consequently, the comparison between the two methods will be made only through the freezing curves.

Ice saturation results from the ultrasonic experiments are presented in figures $6 \mathrm{~A}, 7 \mathrm{~A}$ and $8 \mathrm{~A}$, while those from the capacitive method are shown in figures $6 \mathrm{~B}, 7 \mathrm{~B}$ and $8 \mathrm{~B}$. The curves 
obtained from the ultrasonic apparatus and the capacitive one appear to be consistent: For Sintered glass beads, the whole porous network freezes and thaws in a similar way at $0^{\circ} \mathrm{C}$. This behavior is not surprising since the sintered glass beads consist of a material with a monodisperse micrometric porosity. For Boom Clay, an important freezing-thawing peak is observed near $-4^{\circ} \mathrm{C}$. Then saturation varies almost linearly with temperature until $-80^{\circ} \mathrm{C}$. Moreover likewise the SBG experiments, freezing and thawing behavior are nearly similar. Finally the hardened cement paste experiments exhibit small ice formation until $-30^{\circ} \mathrm{C}$, followed by a more important linearly variation of $S_{c}$ until $-90^{\circ} \mathrm{C}$ while the thawing peaks are respectively around $-60^{\circ} \mathrm{C}$ and $0^{\circ} \mathrm{C}$. It should be emphasized that, unlike in the SBG and BC experiments, a hysteresis loop is observed in the $S_{c}-T$ curve of the hardened cement paste. So that the link between the temperature and the saturation degree cannot reduce, in this case, to a one-to-one relation.

The quantitative comparison between the ice saturation values obtained by ultrasonic measurements $\left(S_{c}^{U}\right)$ and capacitive measurements $\left(S_{c}^{C}\right)$ is given in table 2. As we can see results obtained from the two methods appear to be very close. Indeed, the $\left|S_{c}^{C}-S_{c}^{U}\right|$ difference remains lower than 0.06 which is of order of magnitude of the accuracy of the measurement. Moreover, in the case of hardened cement pastes, the capacitive and the ultrasonic methods provide respectively at $-30^{\circ} \mathrm{C} S_{c}$ equal to 0.22 and 0.16 , which is in good agreement with the value of $S_{c}=0.22$ obtained at the same temperature by low temperature calorimetry (Badger and Sellevold 1986). In conclusion, the two apparatus presented in this study can predict with a good accuracy the amount of the ice formed within a consolidated porous medium. Consequently they represent cheaper alternatives to the low temperature calorimetry.

\section{ICE SATURATION CURVE AND POROUS NETWORK CHARACTERIZATION}

As observed in the previous experimental results, water does not solidify in the whole porous volume at the same temperature: freezing occurs progressively as the temperature is reduced. Once formed somewhere, ice cannot propragate instantaneously through the whole 
material because of the Thomson-Gibbs law and its porous microstructure features (such as the pore sizes distribution - from $\mathrm{nm}$ to micrometers at least - and geometry). In the following, we present how the experimentally-determined function $S_{c}(T)$, giving the amount of ice versus the temperature, can be used with the Thomson-Gibbs law to obtain such microstructural information.

\section{Thomson-Gibbs law}

The Thomson-Gibbs law (Brun et al. 1977; Scherer 1993; Fabbri et al. 2006) comes from the solid-liquid equilibrium (described by the chemical potentials) and from the mechanical equilibrium of a curved interface (Young-Laplace law), which is presented below.

Let $\mu_{J}$ be the chemical potential per mass unit of phase $J=l$ (for liquid water) or $c$ (for ice crystal $)$ and $T\left(T[\mathrm{~K}]=\theta\left[{ }^{0} \mathrm{C}\right]+273.15\right)$ the absolute temperature. The thermodynamic equilibrium between water in liquid form (index $l$ ) and its ice crystal (index $c$ ) requires the equality of their specific chemical potentials, reading:

$$
\mu_{c}\left(T, \boldsymbol{\sigma}_{c}\right)=\mu_{l}\left(T, p_{l}\right)
$$

where $\boldsymbol{\sigma}_{\boldsymbol{c}}$ and $p_{l}$ are the stress tensor and the pressure of the solid ice and the liquid water, respectively. As indicated in (Scherer 1993; Coussy and Monteiro 2007) the shear contribution in ice can be neglected so that (8) reduces to:

$$
\mu_{c}\left(T, p_{c}\right)=\mu_{l}\left(T, p_{l}\right)
$$

where $p_{c}$ is the pressure in the ice crystal. Hereafter the atmospheric pressure (set equal to zero such that $p_{J}$ refers to relative pressure) is adopted as the common reference pressure and the corresponding freezing point $T_{0}$ as the reference temperature (273.15K for water). From the differentiation of (9), the Gibbs-Duhem relation $\mathrm{d} \mu_{J}=\frac{1}{\rho_{J}} \mathrm{~d} p_{J}-s_{J} \mathrm{~d} T$ furnishes:

$$
\frac{1}{\rho_{c}} \mathrm{~d} p_{c}-s_{c} \mathrm{~d} T=\frac{1}{\rho_{l}} \mathrm{~d} p_{l}-s_{l} \mathrm{~d} T
$$


where $\rho_{J}$ is the mass density which is assumed to be constant $\left(\rho_{c} \simeq 0.9167 \mathrm{~g} / \mathrm{cm}^{3}\right.$ and $\rho_{l} \simeq 0.9998 \mathrm{~g} / \mathrm{cm}^{3}$ (Petrenko and Whitworth 1999)), and $s_{J}$ are the entropy per mass unit of phase $J$. Since the strain latent heat (that is the heat exchanged by the considered system with the outside in an evolution when both temperature and pressure are held constant) and the variation of heat capacity with temperature are negligible, the specific entropy is written as:

$$
s_{J}=s_{J}^{0}+c_{p, J} \ln \frac{T}{T_{0}}
$$

with $s_{J}^{0}$ the bulk specific entropy and $c_{p, J}$ the specific heat capacity, constant and associated with the reference state. Defining the capillary pressure as $p_{c a p}=p_{c}-p_{l}$, integrating (10) from the reference state, and using (11), we derive:

$$
p_{\text {cap }}=S_{f}\left(T_{0}-T\right)+C_{f}\left(T-T_{0}+T \ln \frac{T_{0}}{T}\right)=\mathrm{g}(T)
$$

where $S_{f}=\rho_{c}\left(s_{l}-s_{c}^{0}\right)$ is the entropy of fusion per unit of ice crystal volume, $C_{f}=$ $\rho_{c}\left(c_{p, l}-c_{p, c}\right)$ the heat capacity difference between water and ice per unit of ice crystal volume. Function $\mathrm{g}(\mathrm{T})$ is the state function which links the temperature $T$ and the capillary pressure $p_{\text {cap }}$ once the thermodynamic equilibrium is reached between the liquid water and the ice already formed (Coussy 2005).

The mechanical equilibrium of the ice/water interface (Young-Laplace law) gives:

$$
p_{c a p}=\gamma \kappa
$$

where $\gamma \simeq 36+0.25\left(T-T_{0}\right)$ (Zuber and Marchand 2004) is the water / ice interface energy and $\kappa=\mathrm{d} A_{l c} / \mathrm{d} V_{c}=1 / r_{1}+1 / r_{2}$ the interface curvature where $A_{l c}$ is the water/ice interface area, $V_{c}$ the ice crystal volume and $r_{J=1,2}$ are the principal curvature radii of the ice / water interface. Combination of (12) and (13) gives the Thomson-Gibbs law. 


\section{Thermoporometry and results}

From now, the liquid-ice interface is supposed to be spherical. Thus, we classically have:

$$
\kappa=\frac{2}{r}
$$

where $r$ is the radius of the ice crystal. Assuming that the ice crystal is surrounded by a homogeneous layer of unfrozen water, $r$ is related to the pore radius $r_{p}$ and the layer thickness $e$ that remains constant and equal to $9 \AA$, by the relation (Brun et al. 1977; Dash et al. 1995):

$$
r=r_{p}-e .
$$

The freezing and the melting temperatures $T_{f}^{*}$ and $T_{m}^{*}$, respectively, for an ice crystal just filling the cylindrical pore and in equilibrium with liquid water in adjacent pores, are related to the corresponding pore radius $r_{p}^{f}$ and $r_{p}^{m}$, respectively, from (12) to (15):

$$
T_{i}^{*}=\mathrm{g}^{-1}\left(\frac{2 \gamma}{r_{p}^{i}-e}\right) ; i=f \text { or } m
$$

Based on relation (16), the measurement of the successive freezing or melting temperatures provides a means of assessing the pore radii distribution of a porous material. $S_{c}$ is linked to the volume of ice crystal, $V_{c}$, and the volume of the sample, $V_{s}$, by the relation $S_{c}=V_{c} /\left(\phi_{0} V_{s}\right)$. Assuming spherical pore shape, $V_{c}$ is linked to the volume of the unfrozen pores, $V_{u f}$, by the relation:

$$
\mathrm{d} V_{u f}(T)=-\left(\frac{r_{p}}{r_{p}-e}\right)^{3} \mathrm{~d} V_{c}(T)
$$

Thus, a $S_{c}-T$ curves can be translated to a $V_{u f}-r_{p}$ curve, which leads to the following expression for the logarithmic pore size distribution curve $\varphi\left(r_{p}\right)$ :

$$
\varphi\left(r_{p}\right)=-\frac{\phi_{0}}{\gamma_{d}}\left(\frac{r_{p}}{r_{p}-e}\right)^{3} \frac{\partial S_{c}}{\partial \log \left(r_{p}\right)}
$$


As shown in figure 9, whatever the experimental device used, the $\varphi-r_{p}$ curve of boom clay samples obtained from a freezing and a thawing experiment are consistent and exhibit two porosity peaks: around $2 \mathrm{~nm}$ and around $10 \mathrm{~nm}$. The existence of these two peaks, associated to intra-aggregate porosity, is in good agreement with literature data (Romero et al. 1999).

Thermoporometry results of hardened cement paste samples are shown in figure 10 . Freezing tests lead to a large peak around 1-2 $\mathrm{nm}$ and a smaller one around $50 \mathrm{~nm}$. Thawing tests lead to similar results except that the peaks occur at slightly larger pores radius than for freezing.

Comparisons of the $\varphi-r_{p}$ curves from capacitive measurement, from ultrasonic measurement and from porosimetry intrusion test are shown in figure 11 for Boom clay samples and in figure 12 for hardened cement paste. This comparison was only made for the $\varphi-r_{p}$ curves given by a freezing experiment as the kinetics of thawing for the two methods could not be the same.

For hardened cement paste samples, ultrasonic and capacitive results appear to be close and seem to be complementary with the mercury intrusion porosimetry tests: the $100 \mathrm{~nm}$ one is not observed on thermoporometry curves while only the beginning of the $2 \mathrm{~nm}$ peak is observed on mercury intrusion porosimetry curves. Thus thermoporometry appears to be more suitable for scanning pore radii less than $100 \mathrm{~nm}$ while mercury intrusion porosimetry test is more suitable for scanning pore radii higher than $10 \mathrm{~nm}$.

The same conclusion can be made for Boom clay measurements where ultrasonic and capacitive curves are almost the same: only the $2 \mathrm{~nm}$ and $10 \mathrm{~nm}$ peaks are observed. Conversely, the larger pores are only observed by mercury intrusion porosimetry.

The freezing-thawing temperature was linked to the radius of the pore in which the water or the ice are confined. The pore size distributions obtained from capacitive and ultrasonic measurements are in good agreement with data in the literature and complementary to mercury intrusion porosimetry tests. The next step is to understand the physical meaning 
of the liquid to ice phase change hysteresis and to understand how to link it to the porous network characteristics.

\section{Physical meaning of the water to ice phase change hysteresis}

It is well-known (Scherer 1993; Béjaoui et al. 2002) that a liquid water filled pore of radius $r_{p}$ does not generally freeze at $T_{f}^{*}\left(r_{p}\right)$ due to supercooling (metastable liquid phase). Instead in-pore ice formation results from ice percolation through smaller (nanoscopic) channel-like pores connected to already frozen pores (Scherer 1993) or from nucleation process which can only occur at a temperature lower than $T_{f}\left(r_{p}\right)$ (Corr 2001). Consequently, the liquid water filling a pore with radius $r_{p}$ is likely to freeze $\left(r_{p}>r_{p}^{*}\right)$. If it is connected with almost one frozen channel, its water will freeze, quasi-instantaneously, by propagation. However if it is only connected with ice crystal through a smaller channel (which can be called "percolation channel", $r_{c}<r_{p}^{*}$ ) which is not frozen (figure 13), solidification does not occur. It will happen when the temperature decrease will be large enough to allow propagation of ice through the smaller channel. As nucleation can only occur at temperatures below the equilibrium one, the apparent radius calculated from the measured temperature $\left(r_{f}\right)$ will be smaller than the real one $\left(r_{p}\right)$, so :

$$
r_{c} \leq r_{f} \leq r_{p}
$$

This point can be illustrated by figures 14 and 15 where the temperature evolution of the sample core follows the imposed temperature except around $-5^{\circ} \mathrm{C}$ where a temperature jump is observed. This jump is commonly attributed to the inner massive freezing due to the nucleation process that generates solidification of all the supercooled water contained in the biggest connected pores. After that, no temperature jump is observed until the end of the freezing process. After this inner nucleation process, the ice crystal will progressively grow by propagation through smaller and smaller pores as the temperature decreases.

On the other hand, during heating, thawing appears necessarily at $T_{m}^{*}\left(r_{p}\right)$. This is attributed to existence a thin water film between the crystal and the pore wall which prevents 
from metastability (Dash et al. 1995). Hence, the thawing process is governed by the radius of the pore body. The hysteresis between the freezing pore radius and the thawing pore radius can be explained by a traditional ink-bottle scheme in which $r_{c}$ plays the role of the neck pore radius (Dullien 1992). A polydisperse porous network composed by "big" spherical-like pores connected through small capillaries will engender a great hysteresis while a cyclindrical-like or a thin sheet stacking porous structure should show a reversible behavior between freezing-and thawing.

As shown in figure 2b from (Romero et al. 1999), the Boom clay microstructure exhibits spherical-like inter-aggregate porosity and intra-porosity of a thin sheet stacking structure. However, as it is shown in figure 11, in the tested material, inter-aggregate pores volume appears to be significantly lower than the intra-aggregate ones. In consequence, the lack of hysteresis observed on the Boom clay experiments is consistent with the global thin sheet stacking of the material porous structure.

On the other hand, the hysteresis observed in hardened cement paste samples tests are consistent with their global polydisperse microstructure composed by either spherical-like pores and cylindrical-like nanoscopic tubes (Baroghel-Bouny 1994).

In conclusion, the study of the freezing-thawing hysteresis can provide information on the pore shape: the more pronounced the hysteresis is, the higher the ratio $r_{p} / r_{c}$ is. The importance of this ratio has been already emphasized by recent studies on frost damage (Coussy and Monteiro 2007) and salt crystallization (Coussy 2006).

\section{CONCLUSION}

Dielectric capacitive and ultrasonic apparatus were used to experimentally determine the thermodynamic state relation between the ice volume ratio $S_{c}$ and the temperature. These quantitative methods were successfully applied to samples of sintered glass beads, Boom clay and hardened cement paste. The results obtained by the two methods are found to be in good agreement with data in the literature for hardened cement paste.

Pore radii distribution was similar to the data in the literature and complementary to 
mercury intrusion porosimetry: capacitive and ultrasonic apparatus are more suitable for scanning pore radii less than $100 \mathrm{~nm}$ while the latter is more suitable for scanning pore radii higher than $10 \mathrm{~nm}$. In addition, we succeeded to establish a link between the freezing-thawing hysteresis and the porous network structure.

Therefore we can conclude that the two methods presented here are appropriate to analyze the ice formation in porous samples and to provide insights of their pore structure.

\section{ACKNOWLEDGEMENTS}

We are indebted to Damien Delmoitié and Patrick Zélis for their contribution on dielectric and ultrasonic measurements. We are grateful to Philippe Vié for the fabrication of the sintered glass beads, Alex Bertholet for the set-up of the Ultrasonic device and Eric Dupuis for the development of the data acquisition system.

\section{REFERENCES}

Badger, D. and Sellevold, E. (1986). "Ice formation in hardened cement paste - part I room temperature cured pastes with variable moisture content." Cement and Concrete Research, 16, 709-720.

Bager, D. and Sellevold, E. (1986). "Ice formation in hardened cement paste - part II : Drying and resaturation on room temperature cured pastes." Cement and Concrete Research, 16, $835-844$.

Baroghel-Bouny, V. (1994). "Caractérisation microstructurale et hydrique des pâtes de ciment et des bétons ordinaires et très hautes performances," PhD thesis, École Nationale des Ponts et Chaussées.

Baroghel-Bouny, V. and Chaussadent, T. (1993). "Caractérisation de la texture d'un béton durci partir des isothermes de sorption de vapeur d'eau." Bulletin de Liaison des Laboratoires des Ponts et Chaussées, 187, 69-75.

Béjaoui, S., Revertégat, E., and Bournazel, J. P. (2002). "Mécanismes de formation de la glace au sein des pâtes de ciment et des bétons." Revue Française de Génie Civil, 6, $1309-1332$. 
Brun, M., Lallemand, A., Quinson, J., and Eyraud, C. (1977). "A new method for the simultaneous determination of the size and the shape of pores : The thermoporometry." Thermochimica Acta, 21, 59-88.

Corr, D. J. (2001). "A microscopic study of ice formation and propagation in concrete," PhD thesis, University of California at Berkeley, USA.

Coussy, O. (2005). "Poromechanics of freezing materials." Journal of the Mechanics and Physics of Solids, 53, 1689-1718.

Coussy, O. (2006). "Deformation and stress from in-pore drying-induced crystallization of salt." Journal of the Mechanics and Physics of Solids, 54, 1517-1547.

Coussy, O. and Fen-Chong, T. (2005). "Crystallization, pore relaxation and microcryosuction in cohesive porous materials." Comptes Rendus Mecanique, 333, 507-512.

Coussy, O. and Monteiro, P. (2007). "Unsaturated poroelasticity for crystallization in pores." Computers and Geotechnics, 34, 279-290.

Couvreur, J. and Thimus, J. F. (1996). "Te properties of coupling agents in improving ultrasonic transmission." International Journal of Rock Mechanics and Mining Sciences $\&$ Geomechanics Abstracts, 33, 417-424.

Dash, J. G., Fu, H.-Y., and Wettlaufer, J. S. (1995). "The premelting of ice and its environmental consequences." Reports on Progress in Physics, 58, 115-167.

Dehandschutter, B., Vandycke, S., Vendenberghe, M., Gaviglio, P., Sizun, J., and Wouters, L. (2004). "Microfabric of fractured boom clay at depth: a case study of brittle-ductile transitional clay behaviour." Applied Clay Science, 26(1), 389-401.

Dullien, F. (1992). Pourous Media - Fluid Transport and Pore Structure - Second Edition. Academic Press.

Ellison, W., Lamkaouchi, K., and Moreau, J. (1996). "Water: a dielectric reference." Journal of Molecular Liquids, 68, 171-279.

Fabbri, A. (2006). "Physico-mécanique des matériaux cimentaires soumis au gel/dégel," PhD thesis, Université Paris-Est. 
Fabbri, A., Coussy, O., Fen-Chong, T., and Monteiro, P. J. M. (2008). "Are deicing salts necessary to promote scaling in concrete?." Journal of Engineering Mechanics, In Press.

Fabbri, A., Fen-Chong, T., and Coussy, O. (2006). "Dielectric capacity, liquid water content, and pore structure of thawing-freezing materials." Cold Region Science and Technology, $44,52-66$.

Fen-Chong, T. and Fabbri, A. (2005). "Freezing and thawing porous media : Experimental study with a dielectric capacitive method." Comptes Rendus Mecanique, 333, 425-430.

Fen-Chong, T., Fabbri, A., Guilbaud, J.-P., and Coussy, O. (2004). "Determination of liquid water content and dielectric constant in porous media by the capacitive method." Comptes Rendus Mecanique, 332, 639-645.

Kaatze, U. (1997). "The dielectric properties of water in its different states of interaction." Journal of Solution Chemistry, 26(11), 1049-1112.

Mercury, L., Vieillard, P., and Tardy, Y. (2001). "Thermodynamics of ice polymorphs and ice-like water in hydrates and hydroxydes." Applied Geochemistry, 16, 161-181.

Petrenko, V. F. and Whitworth, R. W. (1999). Physics of Ice. Oxford University Press.

Pigeon, M. (1984). "Microstructure et résistance au gel des ciments et bétons," PhD thesis, Université Pierre et Marie Curie.

Romero, E., Gens, A., and Lloret, A. (1999). "Water permeability, water retention and microstructure of unsaturated compacted boom clay." Engineering Geology, 54, 117-127.

Scherer, G. (1993). "Freezing gels." Journal of Non Crystalline Solids, 155, 1-25.

Thimus, J. F., Aguirre-Puente, J., and Cohen-Tenoudji, F. (1991). "Determination of unfrozen water content of an overconsolidated clay down to $-160^{\circ} \mathrm{c}$ by sonic approaches comparison with classical methods." Ground Freezing 91, Yu and Wang, eds., Balkema, Rotterdam, 83-88.

Thimus, J. F., Aguirre-Puente, J., Cohen-Tenoudji, F., and Leclaire, P. (1993). "Ultrasonic measurements in frozen soils to $-120^{\circ} \mathrm{C}$ : results and interpretation using different theoretical models." Sixth International Conference on Permafrost, Beijing, China. Southern 
China University Technology Press, 611-616.

Timur, A. (1968). "Velocity of compressional waves in porous media at permafrost temperatures." Geophysics, 33, 584-595.

Wang, K., Monteiro, P., Rubinsky, B., and Arav, A. (1996). "Microscopic study of ice propagation in concrete." ACI Materials Journal, 93, 370-377.

Zakri, T., Laurent, J.-P., and Vauclin, M. (1998). "Theoretical evidence for Lichtenecker's mixture formulae based on the effective medium theory." Journal of Physics D: Applied Physics, 31, 1589-1594.

Zuber, B. and Marchand, J. (2004). "Predicting the volume instability of hydrated cement systems upon freezing using poro-mechanics and local phase equilibra." Concrete Science Engineering, 37(268), 257-270. 


\section{List of Tables}

1 Main characteristics of tested materials. The temperature $T$ is in ${ }^{\circ} \mathrm{C}$. . . . $\quad 20$

2 Amount of unfrozen water estimated by the capacitive method of the ultrasonic method. . . . . . . . . . . . . . . . . . . . . 21 


\begin{tabular}{|l|l|l|l|}
\hline & BC & HCP & SBG \\
\hline$D_{p}[\mu \mathrm{m}]$ & $0.15,2$ and 300 & 0.004 and 0.1 & 30 \\
\hline$\omega[\%]$ & 23 & 23 & 19 \\
\hline$\phi_{0}[\%]$ & 39 & 38 & 30 \\
\hline$\gamma_{d}(\mathrm{~g} / \mathrm{mL})$ & 23.2 & 22.6 & 19.4 \\
\hline$a(\mathrm{~m} / \mathrm{s})$ & -1881 & -14698 & -3460 \\
\hline$b(-)$ & 1.47 & 3.87 & 1.38 \\
\hline$\varepsilon_{m}$ & $0.0041 T+4.73$ & $0.0313 T+15.71$ & $0.0044 T+4.80$ \\
\hline$\varepsilon_{l}$ & $2.9312 T+172.33$ & $0.6154 T+49.41$ & $-0.7542 T+93.35$ \\
\hline
\end{tabular}

TABLE 1. Main characteristics of tested materials. The temperature $T$ is in ${ }^{\circ} \mathbf{C}$. 


\begin{tabular}{|c|cc|cc|}
\multirow{2}{*}{$\theta\left[{ }^{\circ} \mathrm{C}\right]$} & \multicolumn{2}{|c|}{ BC } & \multicolumn{2}{c|}{ HCP } \\
\cline { 2 - 5 } & $S_{c}^{C}$ & $S_{c}^{U}$ & $S_{c}^{C}$ & $S_{c}^{U}$ \\
\hline$-5^{\circ} \mathrm{C}$ & 0.38 & 0.44 & 0.01 & 0.04 \\
$-15^{\circ} \mathrm{C}$ & 0.60 & 0.66 & 0.05 & 0.06 \\
$-25^{\circ} \mathrm{C}$ & 0.71 & 0.74 & 0.13 & 0.11 \\
$-30^{\circ} \mathrm{C}$ & 0.75 & 0.77 & 0.22 & 0.16 \\
$-40^{\circ} \mathrm{C}$ & 0.82 & 0.82 & - & - \\
\hline
\end{tabular}

TABLE 2. Amount of unfrozen water estimated by the capacitive method of the ultrasonic method. 


\section{List of Figures}

1 Pore size distribution in boom clay and hardened cement paste, from mercury intrusion experiments. . . . . . . . . . . . . . . . . . .

2 Temperature dependence of the in-pore liquid dielectric constant in sintered glass beads, boom clay, and hardened cement paste. . . . . . . . . . . . .

3 Temperature dependence of the dielectric constant of the mineral solid in sintered glass beads, boom clay, and hardened cement paste. . . . . . . . 25

4 Schematic representation of the capacitive and the ultrasonic devices. . . . . 26

5 Temperature cycle used for the ultrasonic and the capacitive experiments. . . 27

$6 \quad$ Variation of ice saturation during a freeze-thaw cycle of a specimen of sintered glass beads, evaluated with the ultrasonic apparatus (A) and the capacitive apparatus (B). . . . . . . . . . . . . . . . . .

$7 \quad$ Variation of ice saturation during a freeze-thaw cycle of a specimen of boom clay, evaluated with the ultrasonic apparatus (A) and the capacitive apparatus (B). . . . . . . . . . . . . . . . . . . .

8 Variation of ice saturation during a freeze-thaw cycle of a specimen of hardened cement paste, evaluated with the ultrasonic apparatus (A) and the capacitive apparatus (B) . . . . . . . . . . . . . . .

9 Pore size distribution in a Boom clay sample subjected to freezing and thawing, from capacitive (A) and ultrasonic (B) measurements. . . . . . . . . . . 31

10 Pore size distribution in a hardened cement paste sample subjected to freezing and thawing, from capacitive (A) and ultrasonic (B) measurements. . . . . .

11 Comparison between the pore size distribution in a boom clay sample, from the capacitive, ultrasonic and mercury intrusion measurements. . . . . . . . 33

12 Comparison between the pore size distribution in a hardened cement paste sample, from the capacitive, ultrasonic and mercury intrusion measurements.

13 Schematic representation of an unfrozen pore connected to a frozen one by a small capillary channel. . . . . . . . . . . . . . . . . . . . .

14 Core temperature measured by the ultrasonic apparatus during the freezing process. . . . . . . . . . . . . . . . .

15 Core temperature measured by the capacitive apparatus during the freezing process. 


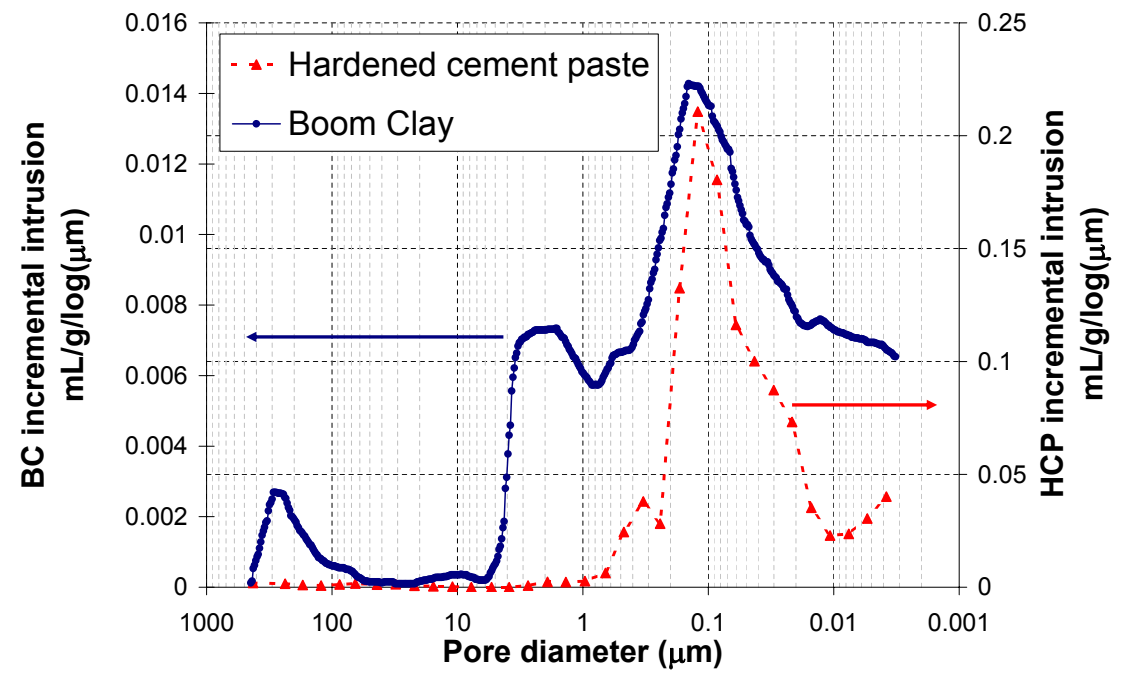

FIG. 1. Pore size distribution in boom clay and hardened cement paste, from mercury intrusion experiments. 


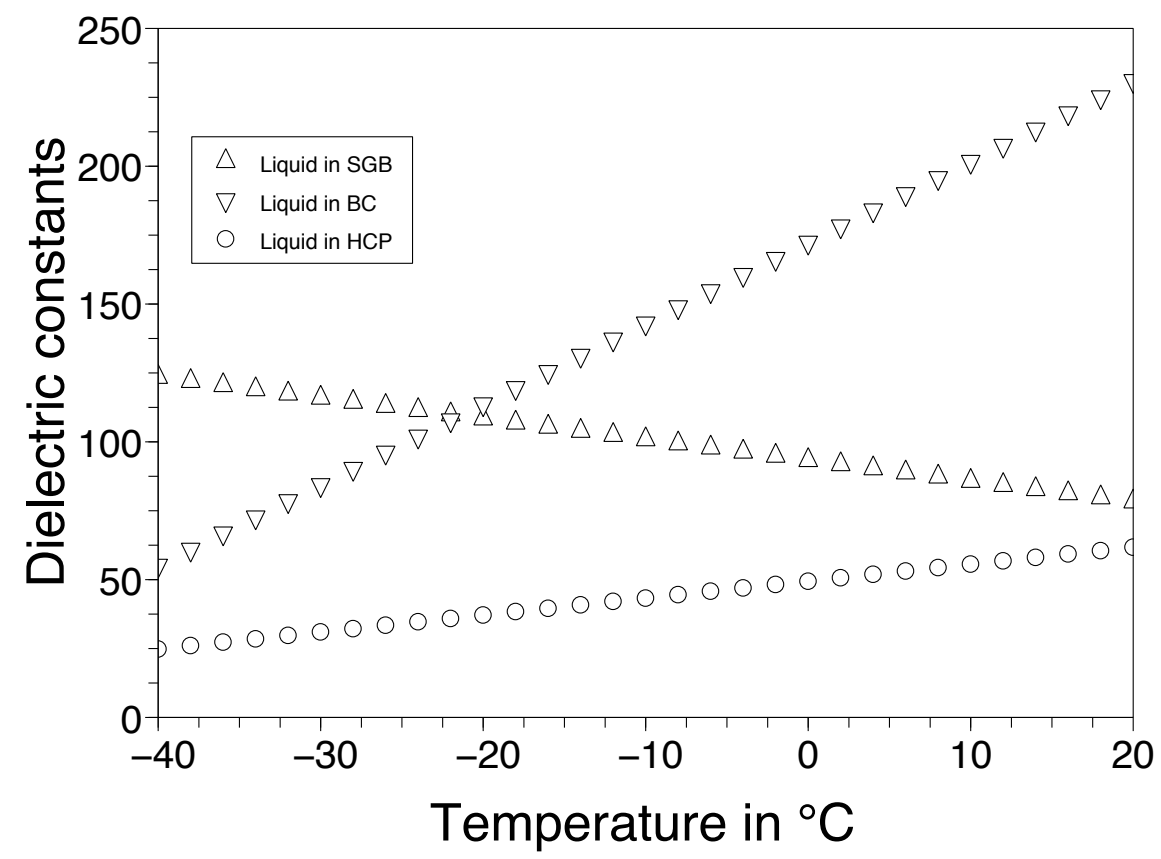

FIG. 2. Temperature dependence of the in-pore liquid dielectric constant in sintered glass beads, boom clay, and hardened cement paste. 


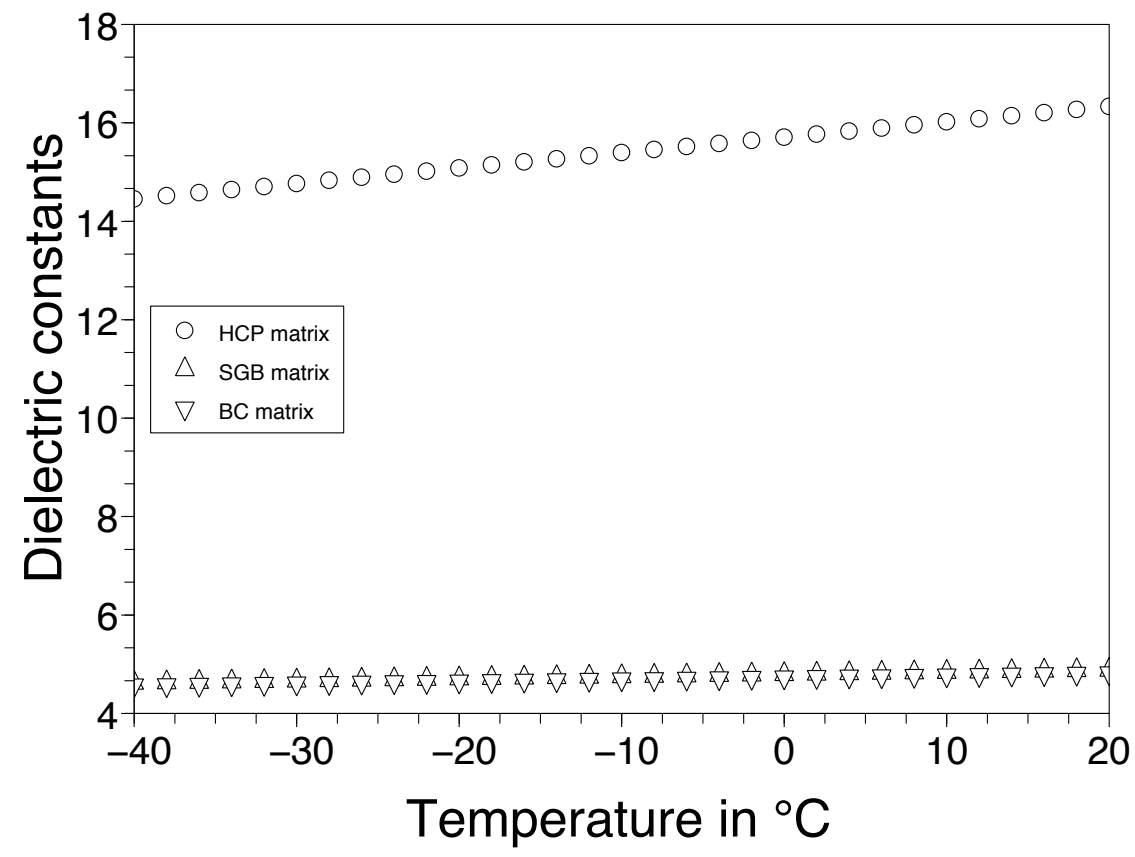

FIG. 3. Temperature dependence of the dielectric constant of the mineral solid in sintered glass beads, boom clay, and hardened cement paste. 


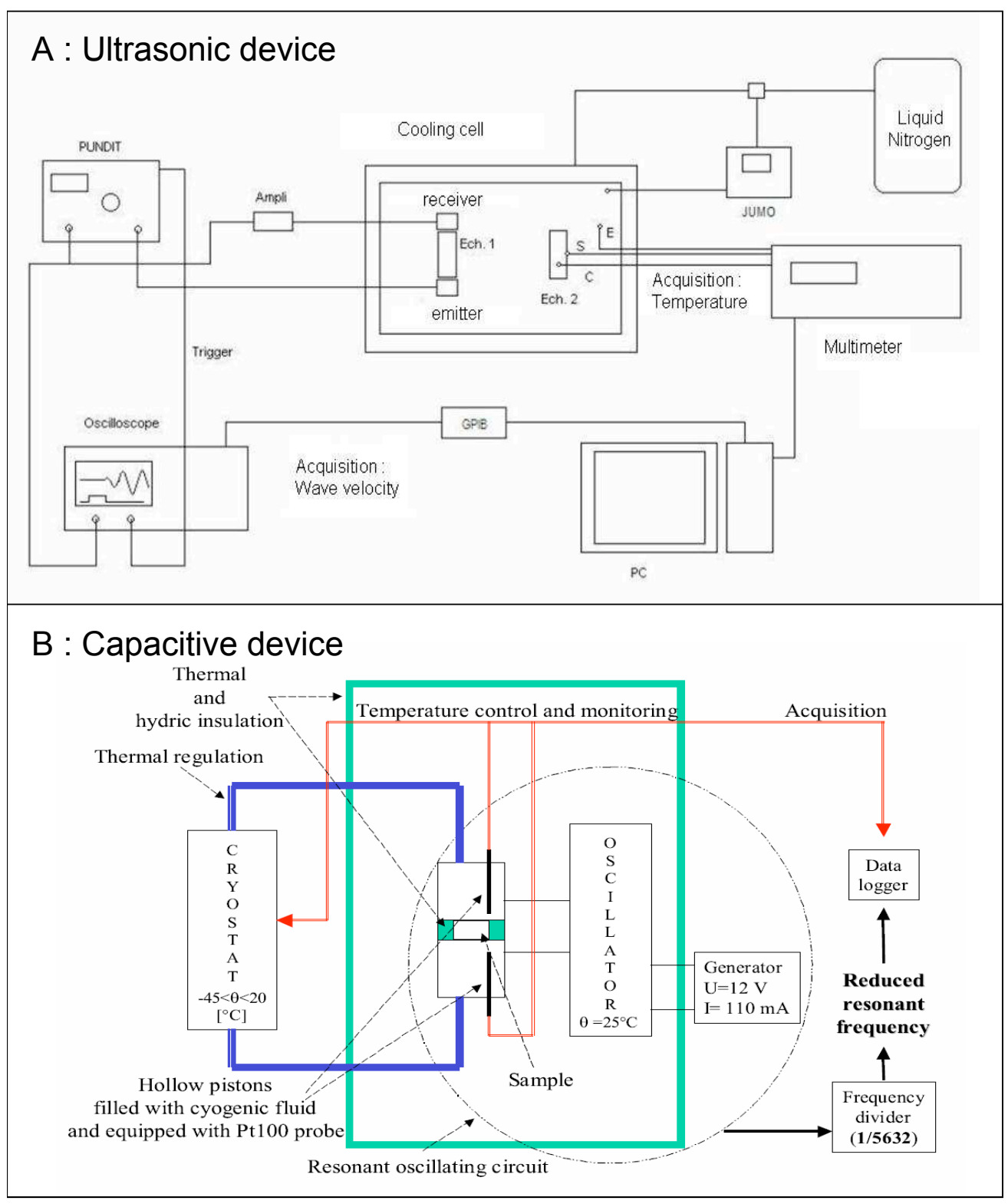

FIG. 4. Schematic representation of the capacitive and the ultrasonic devices. 


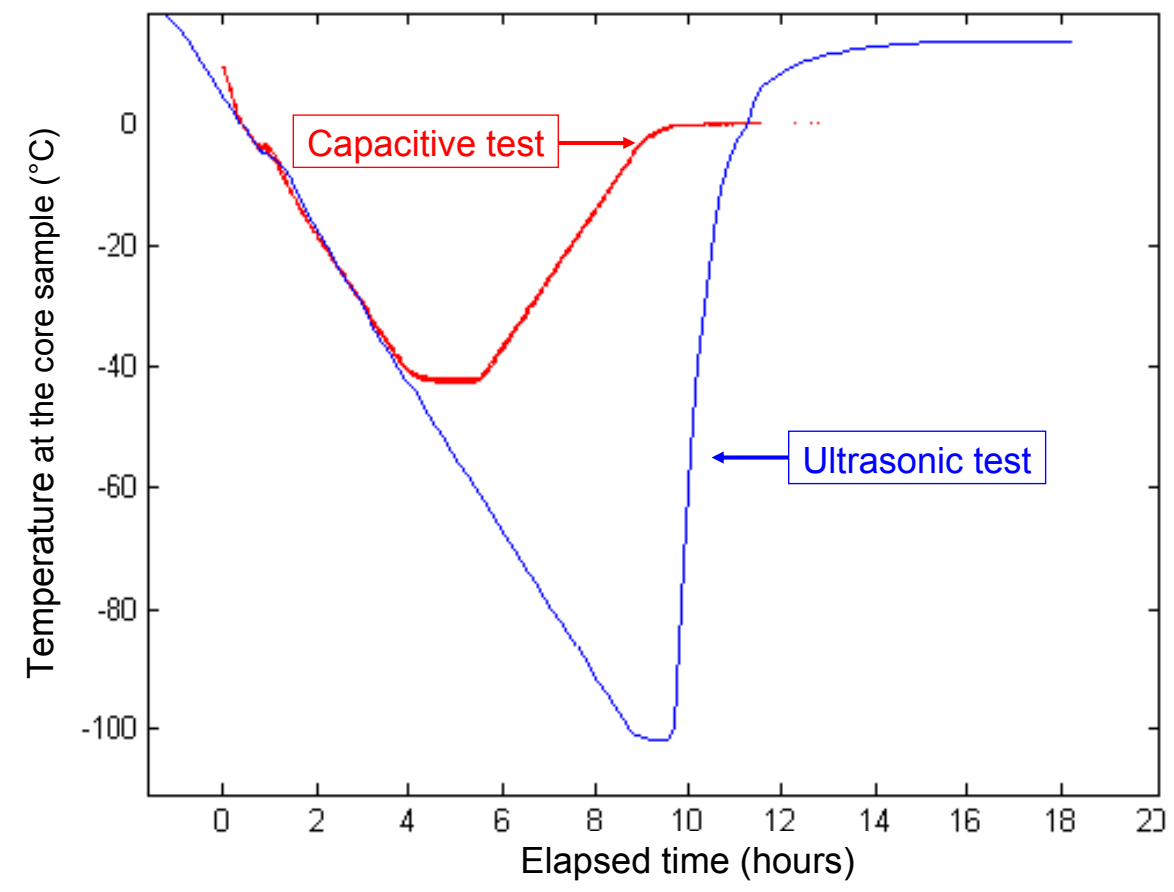

FIG. 5. Temperature cycle used for the ultrasonic and the capacitive experiments. 

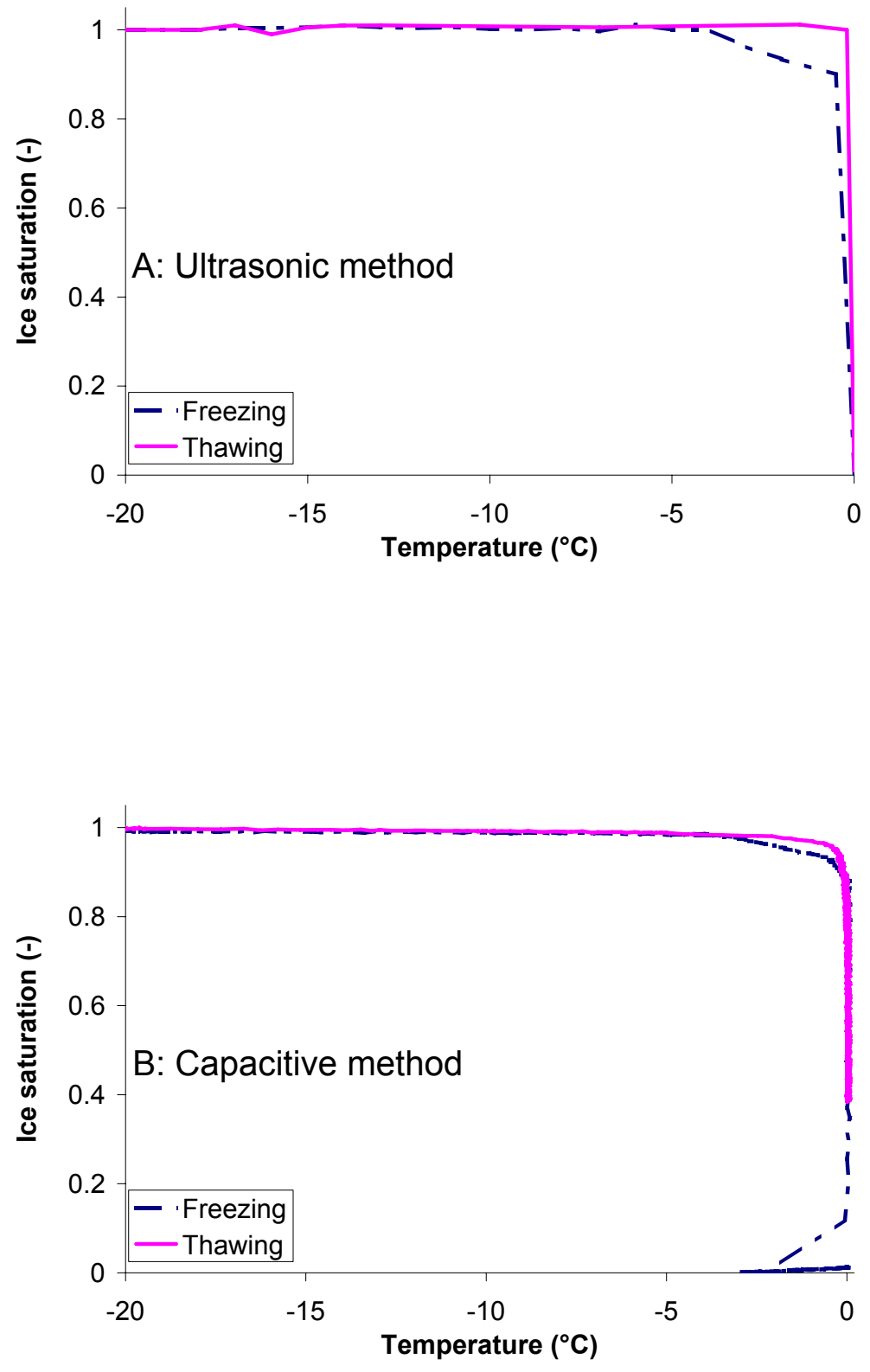

FIG. 6. Variation of ice saturation during a freeze-thaw cycle of a specimen of sintered glass beads, evaluated with the ultrasonic apparatus $(A)$ and the capacitive apparatus (B). 

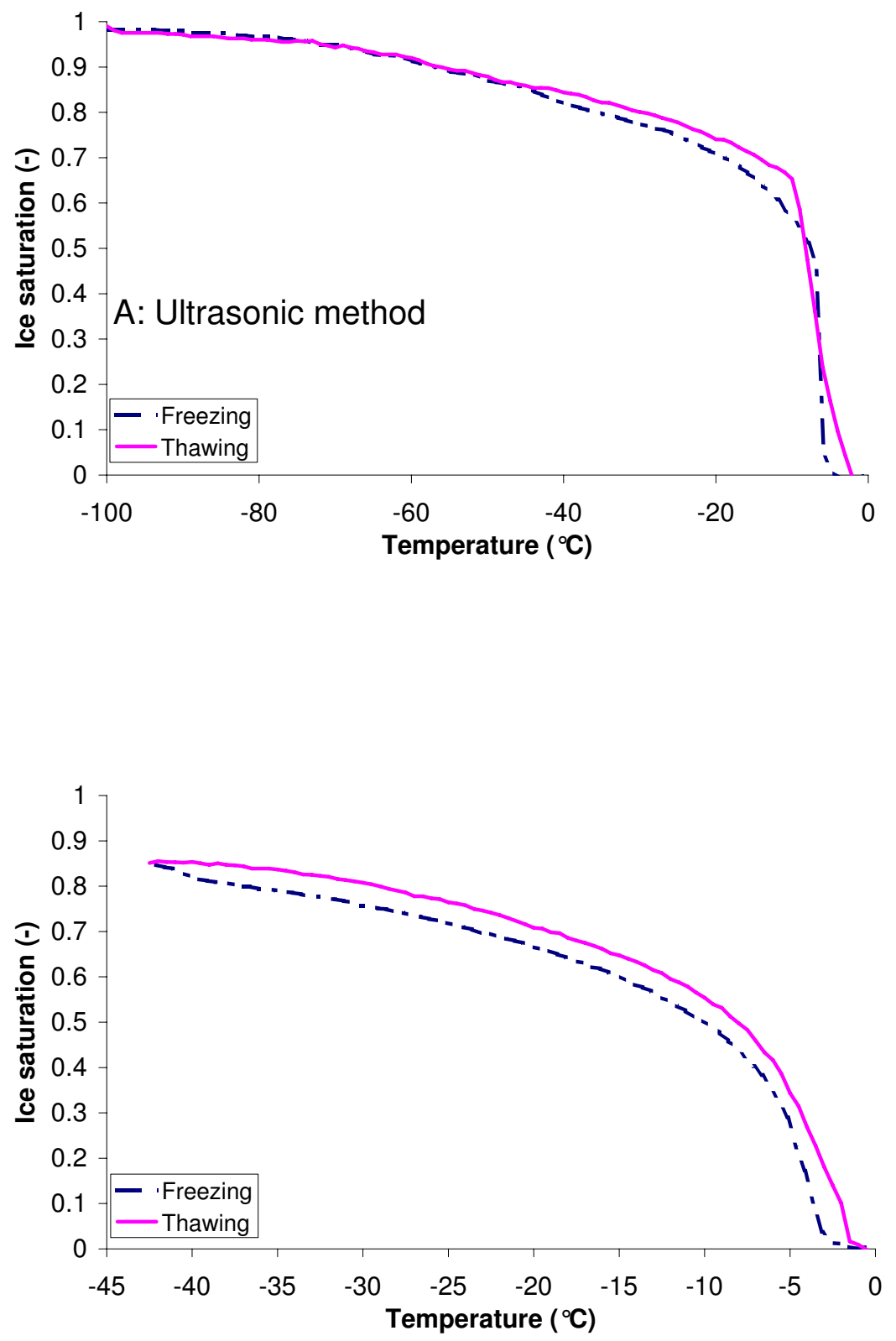

FIG. 7. Variation of ice saturation during a freeze-thaw cycle of a specimen of boom clay, evaluated with the ultrasonic apparatus (A) and the capacitive apparatus (B). 

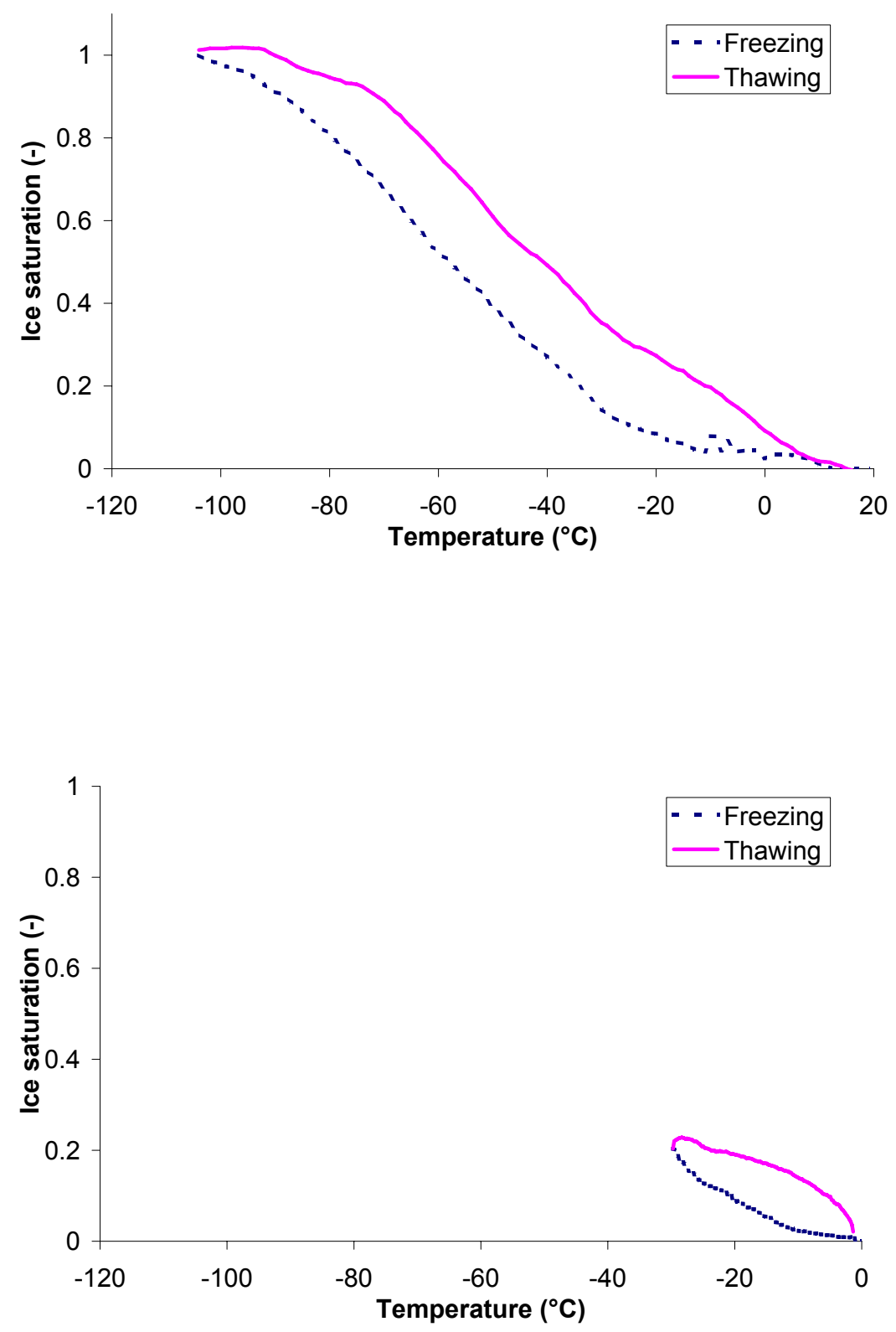

FIG. 8. Variation of ice saturation during a freeze-thaw cycle of a specimen of hardened cement paste, evaluated with the ultrasonic apparatus $(A)$ and the capacitive apparatus (B). 


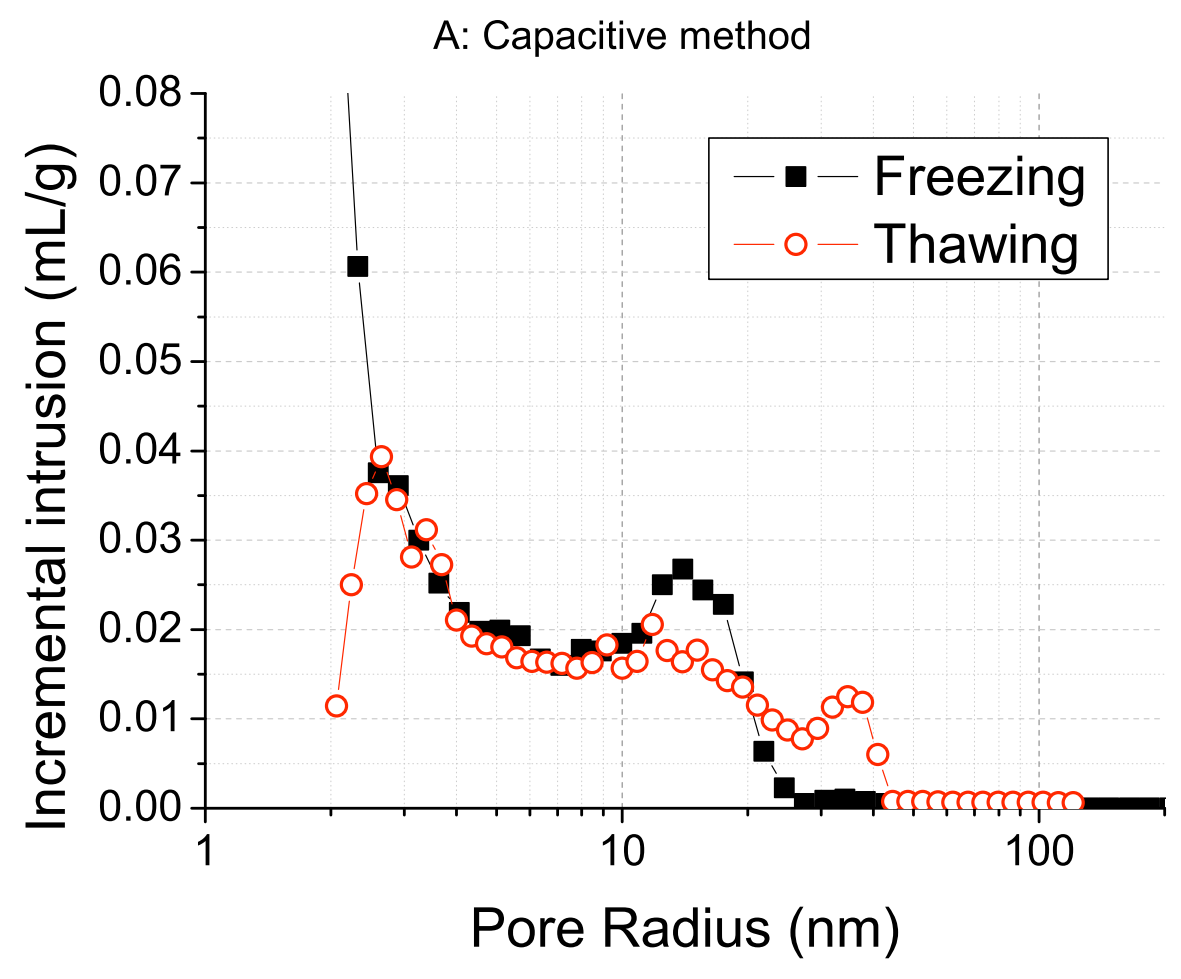

B: Ultrasonic method

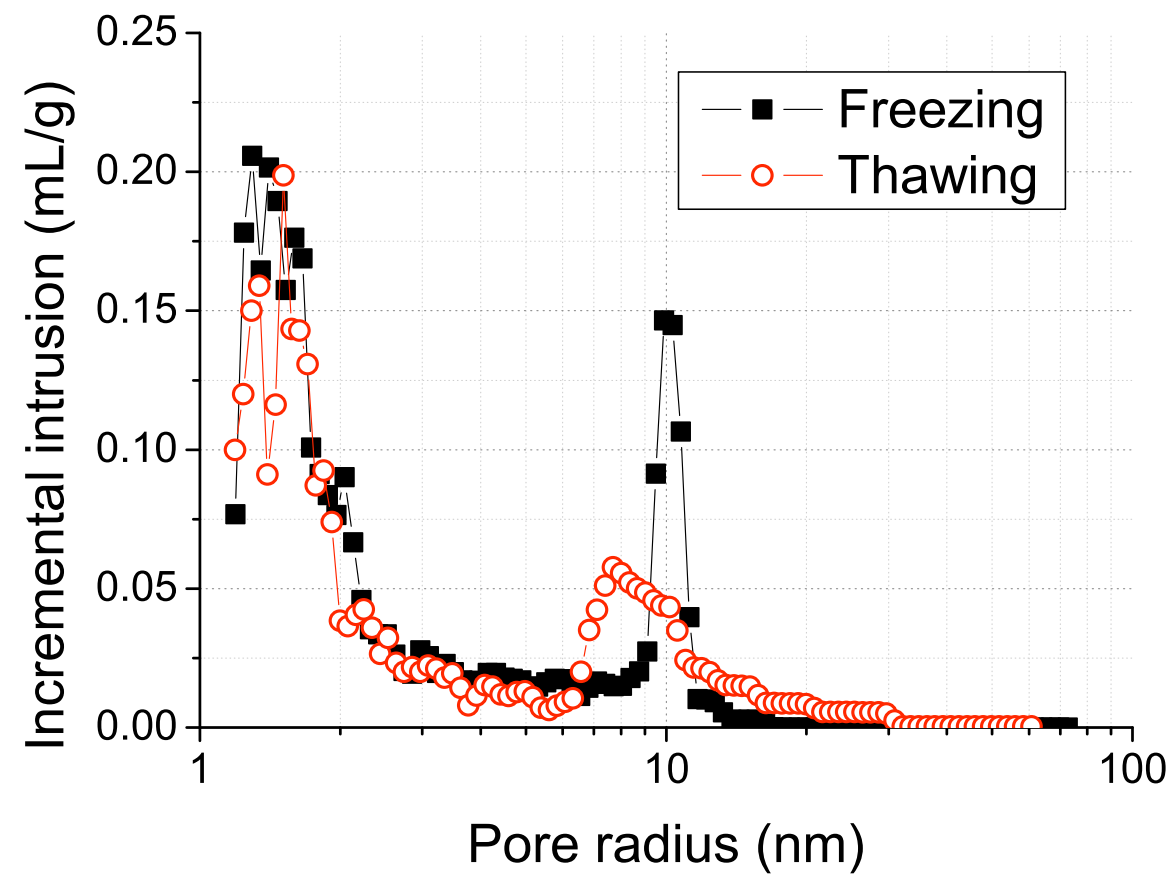

FIG. 9. Pore size distribution in a Boom clay sample subjected to freezing and thawing, from capacitive $(A)$ and ultrasonic (B) measurements. 
A: Capacitive method

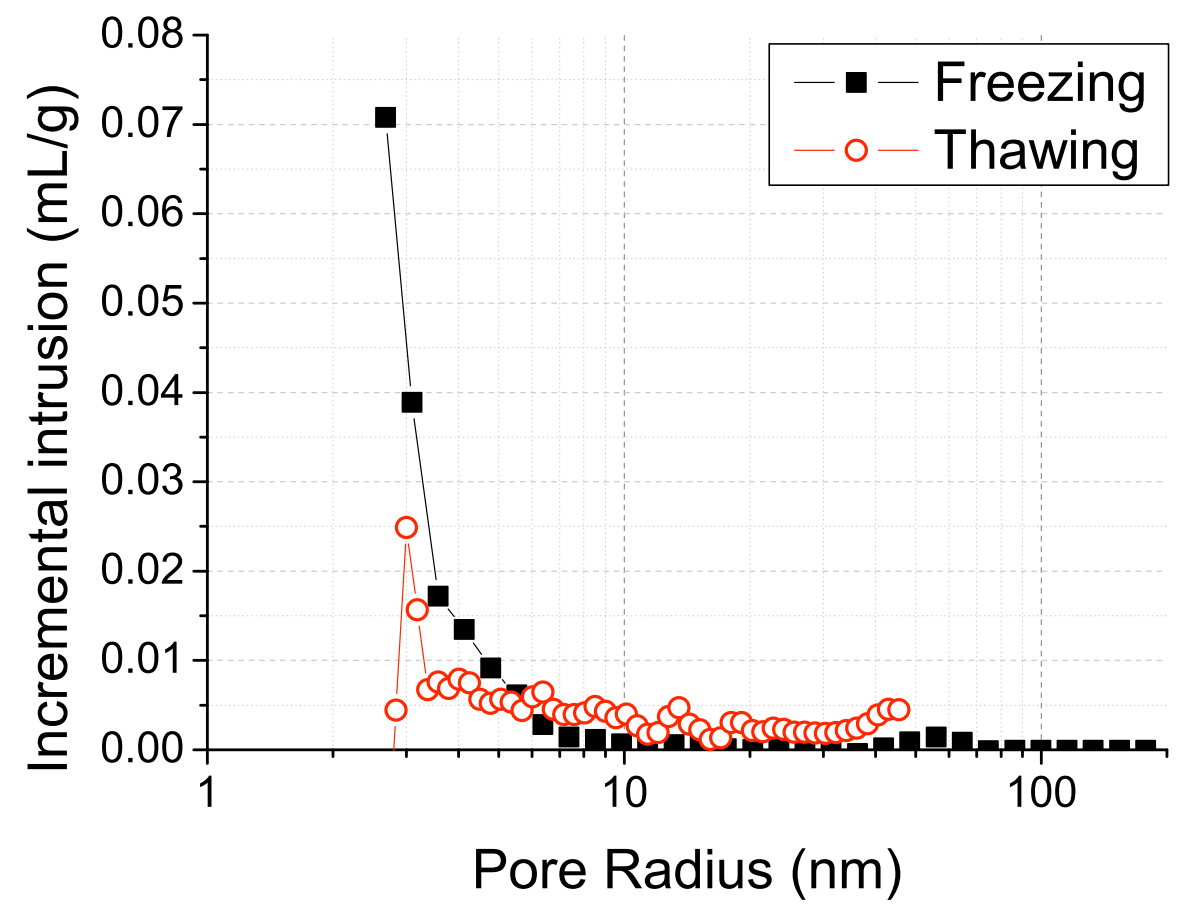

B: Ultrasonic method

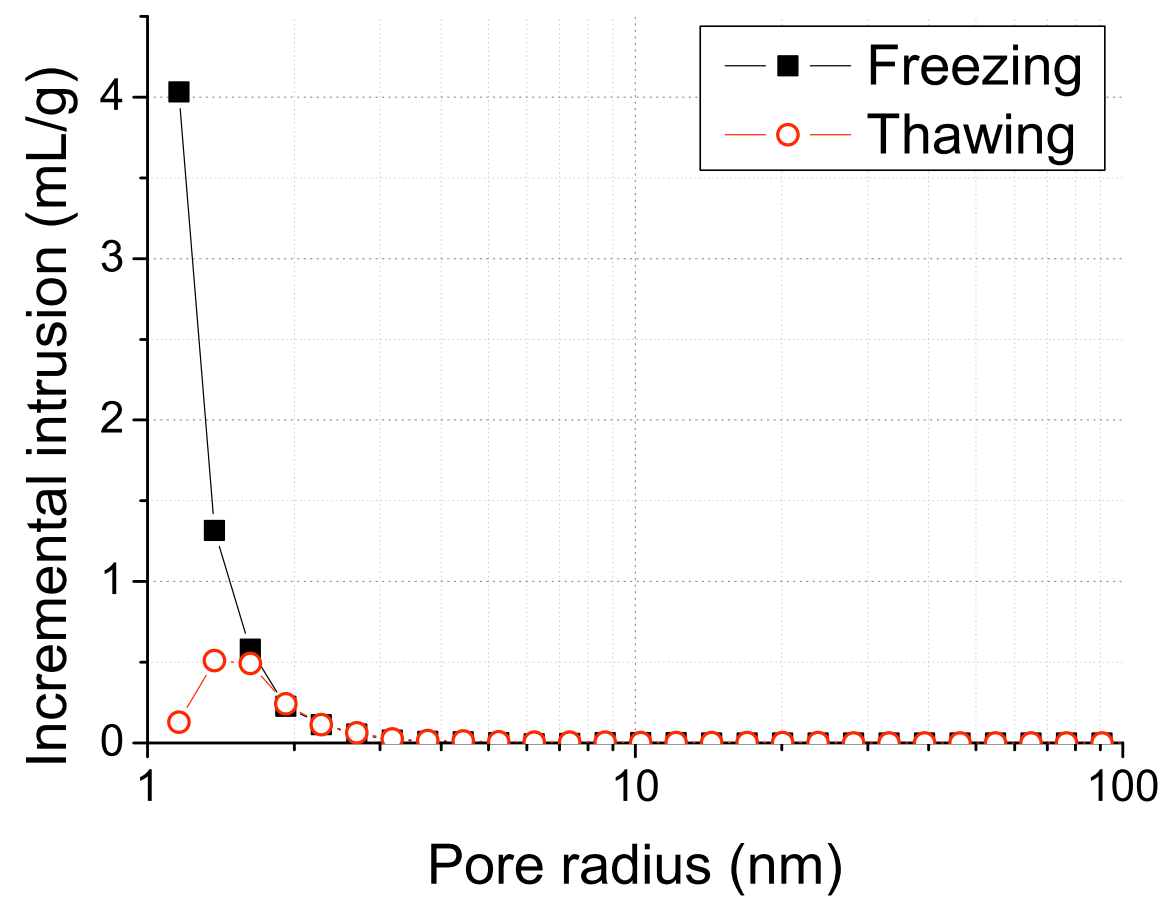

FIG. 10. Pore size distribution in a hardened cement paste sample subjected to freezing and thawing, from capacitive $(A)$ and ultrasonic (B) measurements. 


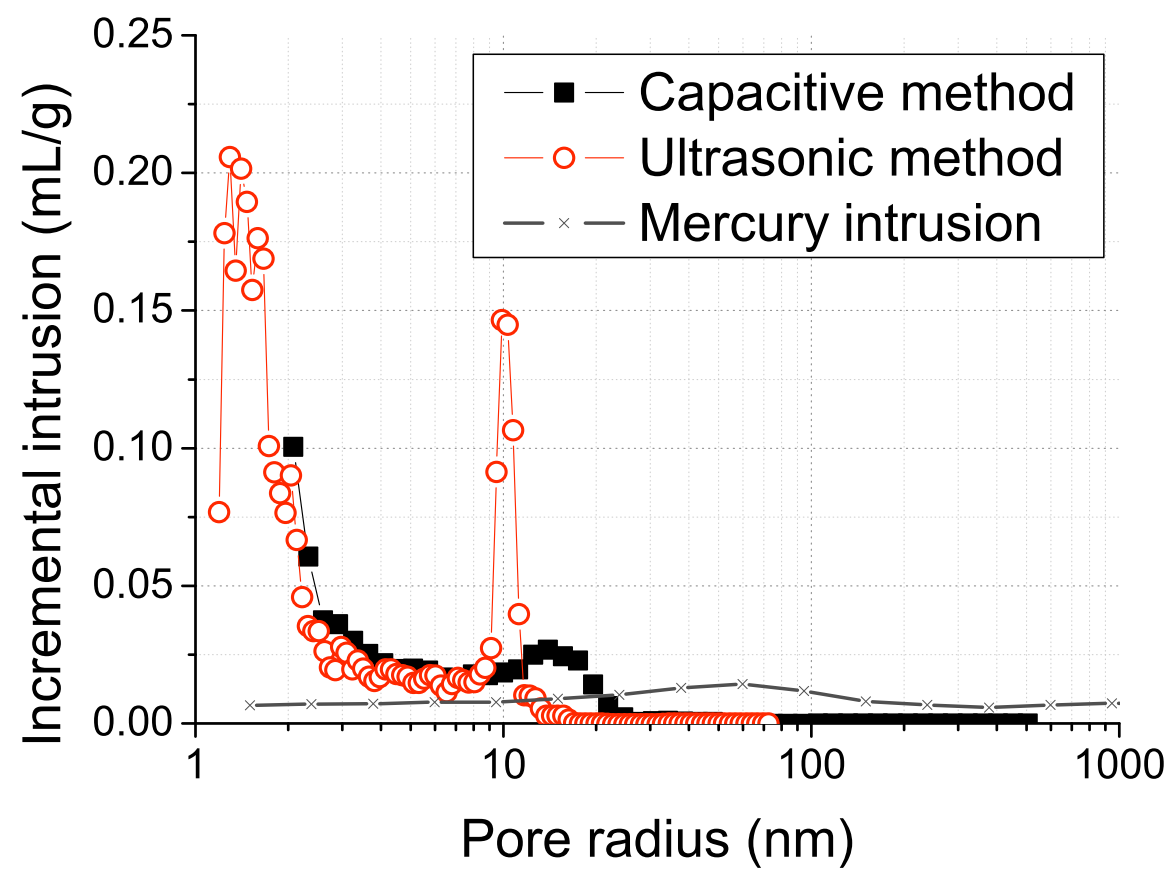

FIG. 11. Comparison between the pore size distribution in a boom clay sample, from the capacitive, ultrasonic and mercury intrusion measurements. 


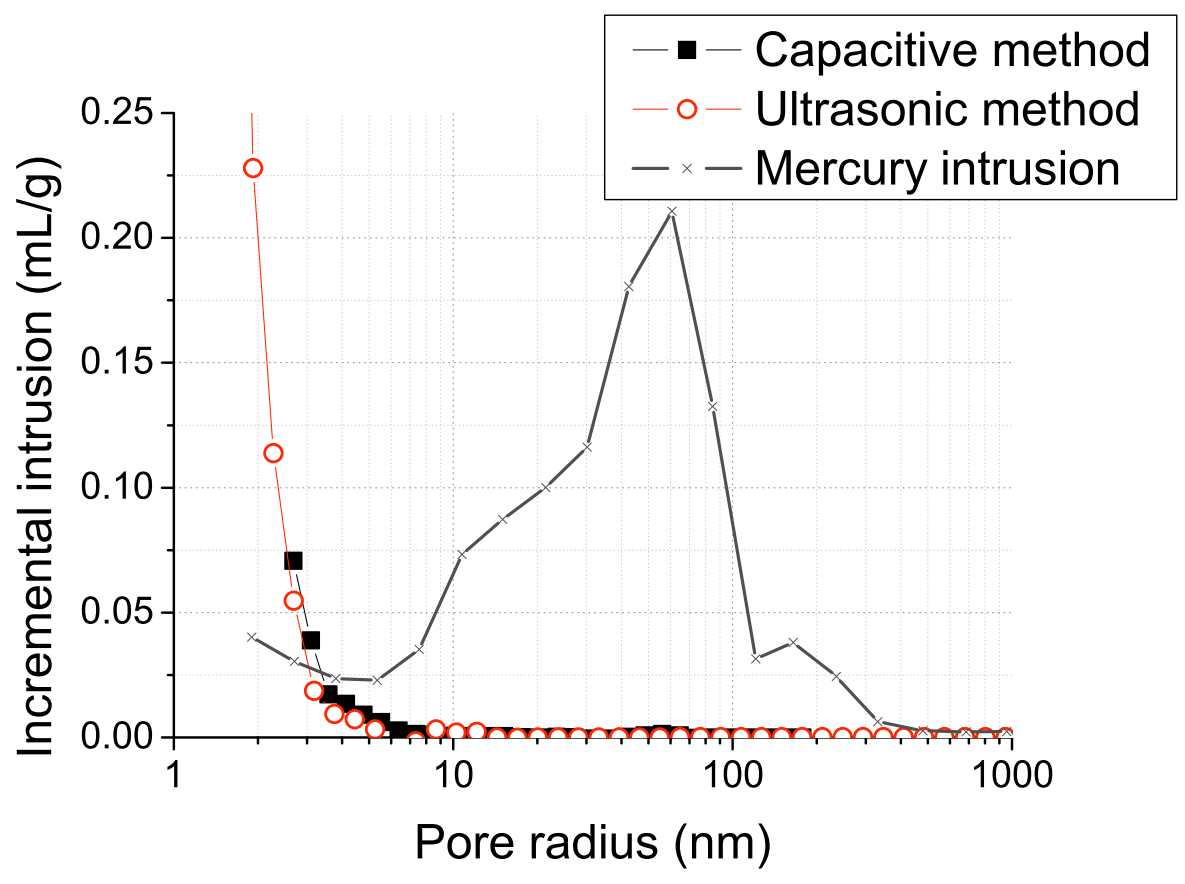

FIG. 12. Comparison between the pore size distribution in a hardened cement paste sample, from the capacitive, ultrasonic and mercury intrusion measurements. 


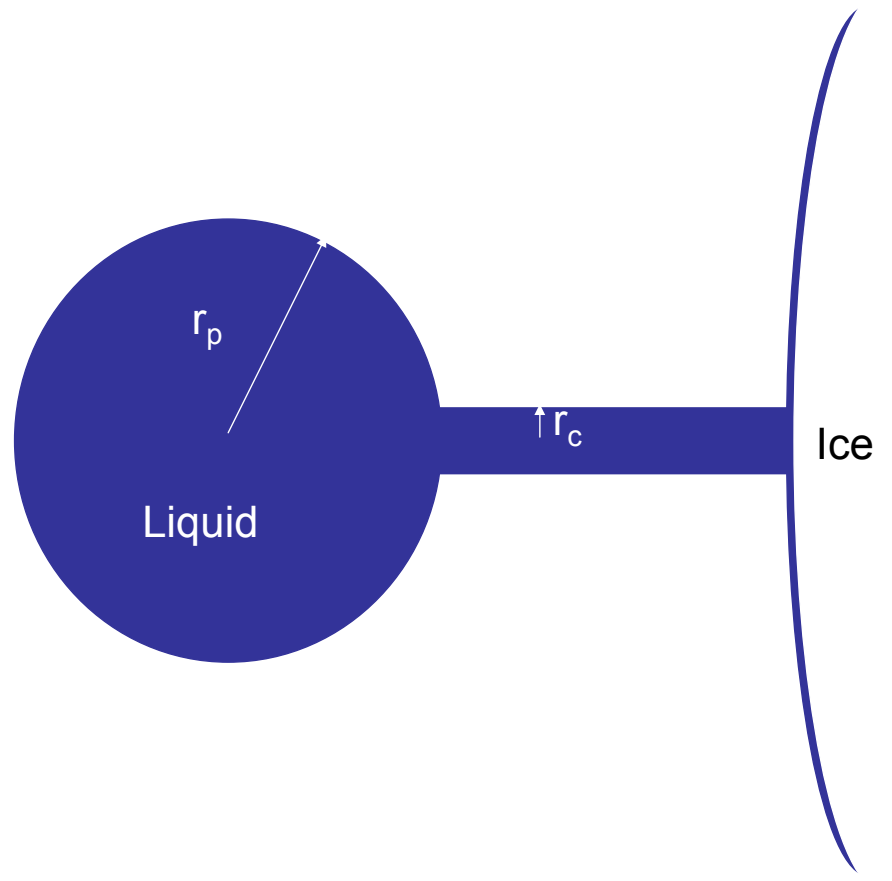

FIG. 13. Schematic representation of an unfrozen pore connected to a frozen one by a small capillary channel. 


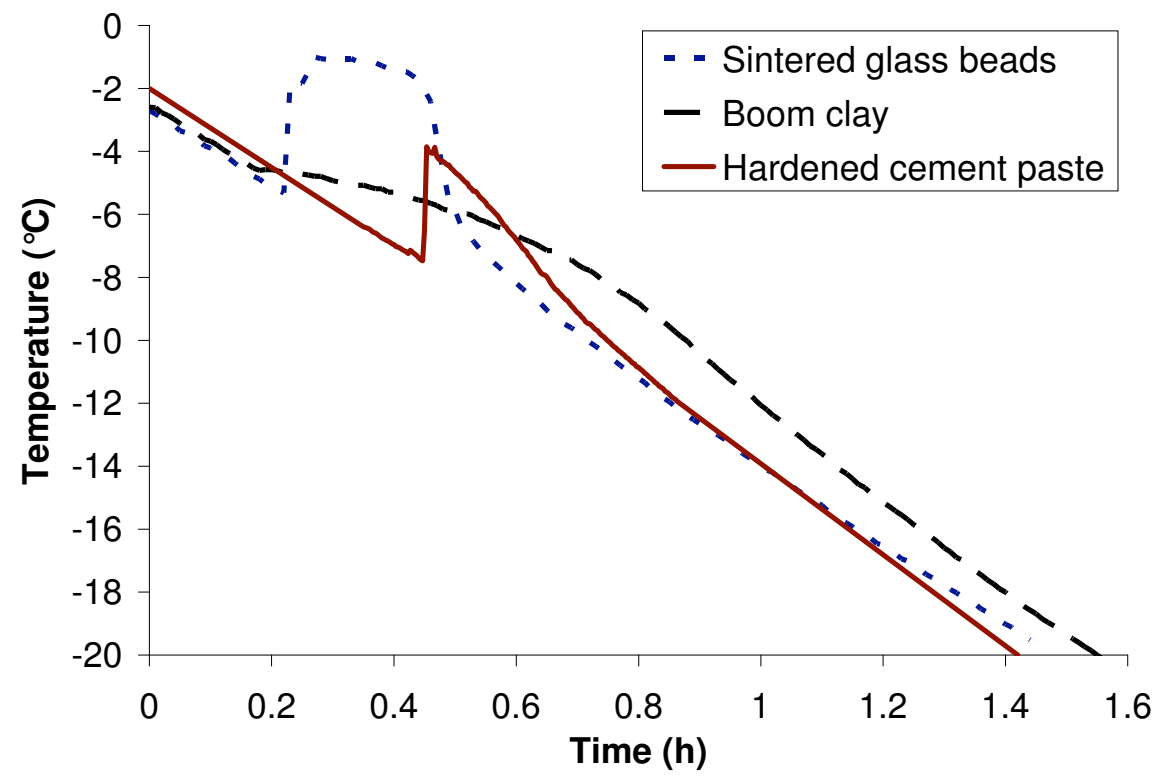

FIG. 14. Core temperature measured by the ultrasonic apparatus during the freezing process. 


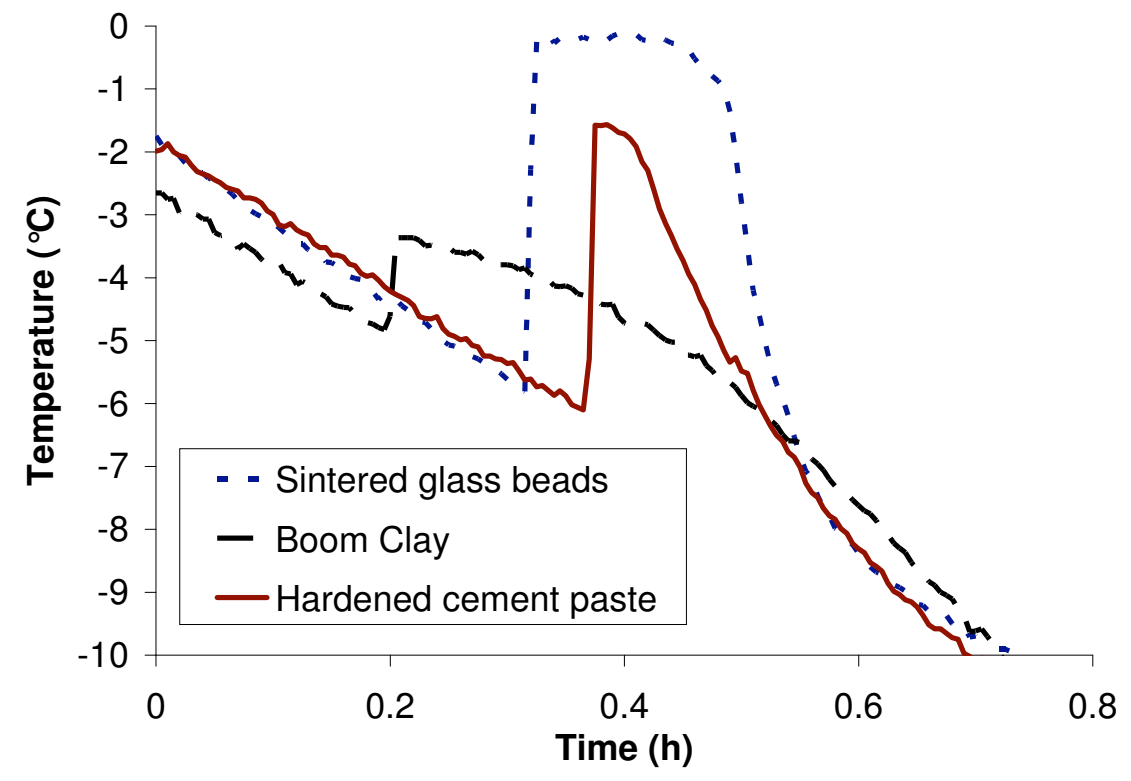

FIG. 15. Core temperature measured by the capacitive apparatus during the freezing process. 